\title{
Asymptotic Analysis of Displaced Lunar Orbits
}

\author{
Jules Simo* and Colin R. McInnes ${ }^{\dagger}$ \\ University of Strathclyde, Glasgow, G1 1XJ, United Kingdom
}

\section{Introduction}

$\mathrm{T}$ HE design of spacecraft trajectories is a crucial task in space mission design. Solar sail technology appears as a promising form of advanced spacecraft propulsion which can enable exciting new spacescience mission concepts such as solar system exploration and deep space observation. Although solar sailing has been considered as a practical means of spacecraft propulsion only relatively recently, the fundamental ideas are by no means new (see McInnes $^{1}$ for a detailed description). A solar sail is propelled by reflecting solar photons and therefore can transform the momentum of the photons into a propulsive force. Solar sails can also be utilised for highly non-Keplerian orbits, such as orbits displaced high above the ecliptic plane (see Waters and McInnes ${ }^{2}$ ). Solar sails are especially suited for such non-Keplerian orbits, since they can apply a propulsive force continuously. In such trajectories, a sail can be used as a communication satellite for high latitudes. For example, the orbital plane of the sail can be displaced above the orbital plane of the Earth, so that the sail can stay fixed above the Earth at some distance, if the orbital periods are equal (see Forward ${ }^{3}$ ). Orbits around the collinear points of the Earth-Moon system are also of great interest because their unique positions are advantageous for several important applications in space mission design (see e.g. Szebehely ${ }^{4}$, Roy, ${ }^{5}$ Vonbun, ${ }^{6}$ Thurman et al. ${ }^{7}$ Gómez et al. ${ }^{8,9}$ ). Several authors have tried to determine more accurate approximations (quasi-Halo orbits) of such equilibrium orbits ${ }^{10}$. These orbits were first studied by Farquhar ${ }^{11}$, Farquhar and Kamel ${ }^{10}$, Breakwell and Brown ${ }^{12}$, Richardson ${ }^{13}$, Howell ${ }^{14,15}$. If an orbit maintains visibility from Earth, a spacecraft on it (near the $L_{2}$ point) can be used to provide communications between the equatorial regions of the Earth and the lunar poles. The establishment of a bridge for radio communications is crucial for forthcoming space missions, which plan to use the lunar poles. McInnes ${ }^{16}$ investigated a new family of displaced solar sail orbits near the Earth-Moon libration points. Displaced orbits have more recently been developed by Ozimek et al. ${ }^{17}$ using collocation methods. In Baoyin and McInnes ${ }^{18,19,20}$ and McInnes ${ }^{16,21}$, the authors describe new orbits which are associated with artificial Lagrange points in the Earth-Sun system. These artificial equilibria have potential applications for future space physics and Earth observation missions. In McInnes and Simmons ${ }^{22}$, the authors investigate large new families of solar sail orbits, such as Sun-centered halo-type trajectories, with the sail executing a circular orbit of a chosen period above the ecliptic plane. We have recently investigated displaced periodic orbits at linear order in the Earth-Moon restricted three-body system, where the third massless body is a solar sail (see Simo and McInnes ${ }^{23}$ ). These highly non-Keplerian orbits are achieved using an extremely small sail acceleration. It was found that for a given displacement distance above/below the Earth-Moon plane it is easier by a factor of order 3.19 to do so at $L_{4} / L_{5}$ compared to $L_{1} / L_{2}$ - ie. for a fixed sail acceleration the displacement distance at $L_{4} / L_{5}$ is greater than that at $L_{1} / L_{2}$. In addition, displaced $L_{4} / L_{5}$ orbits are passively stable, making them more forgiving to sail pointing errors than highly unstable orbits at $L_{1} / L_{2}$. The drawback of the new family of orbits is the increased telecommunications path-length, particularly the Moon- $L_{4}$ distance compared to the Moon- $L_{2}$ distance.

In this note, we study the dynamics of displaced orbits in relation to the two and three-body Earth-Moon problem and compare the results. The solar sail Earth-Moon problem differs greatly from the Earth-Sun system as the Sun-line direction varies continuously in the rotating frame and the equations of motion of the sail are given by a set of nonlinear, non-autonomous ordinary differential equations. Trajectories near the Earth-Moon $L_{1}$ and $L_{2}$ points are not easily identified, such that the solar sail can enable continuous

*Research Fellow, Department of Mechanical Engineering, jules.simo@strath.ac.uk.

†Professor, Department of Mechanical Engineering, colin.mcinnes@strath.ac.uk, Member AIAA. 
communications with the equatorial regions of the Earth from any point on the lunar far-side. We therefore develop an asymptotic analysis for large $\left(a_{0}=1.7 \mathrm{~mm} / \mathrm{s}^{2}\right)$ and small $\left(a_{0}=0.58 \mathrm{~mm} / \mathrm{s}^{2}\right)$ accelerations. This analysis is obtained within an approximation of large displaced orbits $\left(a_{0}=1.7 \mathrm{~mm} / \mathrm{s}^{2}\right)$ by the Moon-Sail two-body problem. The displaced periodic orbits found approach the asymptotic solutions as the characteristic acceleration becomes large. It is shown for example that, with a suitable sail attitude control program, a $4 \times 10^{4} \mathrm{~km}$ displaced, out-of-plane trajectory far from the $L_{2}$ with a sail acceleration of $1.7 \mathrm{mms}^{-2}$ can be approximated using the two-body analysis. This simple, two-body approximate analysis matches with the large displaced orbit found by Ozimek et al. ${ }^{17}$ using numerical collocation methods in a previous study. For small acceleration we use a linear approximation of the Earth-Moon three-body problem which again matches well with Ozimek et al. ${ }^{17}$

\section{Moon-Sail Three-Body Problem}

The motion of a solar sail moving under the gravitational influences of the Earth and the Moon can be described in terms of the circular restricted three-body problem. In this model, we will assume that $m_{1}$ represents the larger primary (Earth), $m_{2}$ the smaller primary (Moon) and we will be concerned with the motion of the sail which has a negligible mass $\left(m_{1}>m_{2}\right)$. It is always assumed that the two more massive bodies (primaries) moving in circular orbits about their common center of mass. If we further restrict the motion of the third body to be in the orbital plane formed by the other two bodies, the problem is the planar circular restricted three-body problem (PCRTBP). In order to develop a mathematical model without loss of generality, it is useful to introduce some parameters that are characteristics of each particular three-body system. This set of parameters is used to normalize the equations of motion. The unit of mass is taken to be the total mass of the system $\left(m_{1}+m_{2}\right)$ and the unit of length is chosen to be the constant separation between $m_{1}$ and $m_{2}$. We further define the time units such that, the orbital period of the primaries about their center of mass is $2 \pi$. Under these considerations the masses of the primaries in the normalized system of units are $m_{1}=1-\mu$ and $m_{2}=\mu$, where

$$
\mu=\frac{m_{2}}{m_{1}+m_{2}}
$$

is the nondimensional gravitational parameter. The geometry for the Earth-Moon restricted three-body system is depicted in Figure 1.

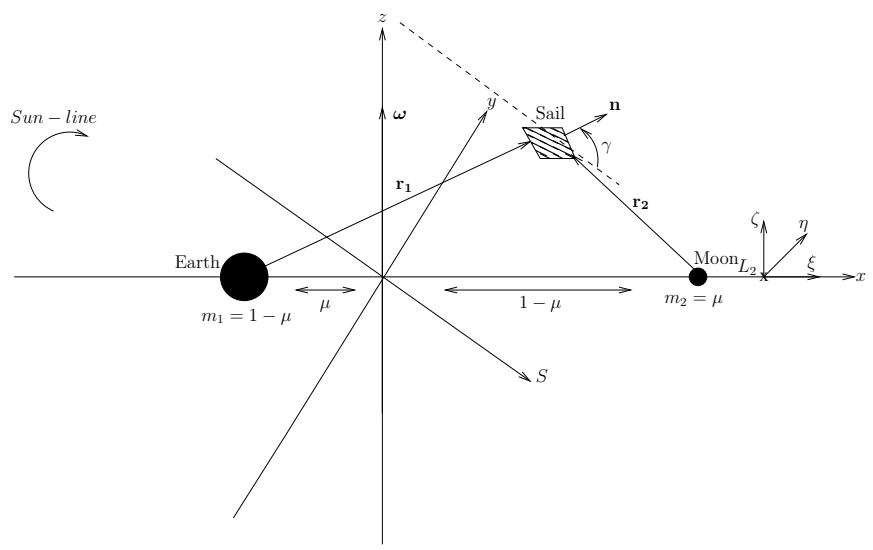

Figure 1. Schematic geometry of the Earth-Moon restricted three-body problem.

\section{II.A. Equations of motion in presence of a solar sail}

The equation for the solar sail in a rotating frame of reference is described by

$$
\frac{d^{2} \boldsymbol{r}}{d t^{2}}+2 \boldsymbol{\omega} \times \frac{d \boldsymbol{r}}{d t}+\nabla U(\boldsymbol{r})=\boldsymbol{a}
$$

where $\boldsymbol{\omega}=\omega \hat{\boldsymbol{z}}(\hat{\boldsymbol{z}}$ is a unit vector pointing in the direction of $\boldsymbol{z}$ ) is the angular velocity vector of the rotating frame and $\boldsymbol{r}$ is the position vector of the solar sail relative to the center of mass of the two primaries. The 
three-body gravitational potential $U(\boldsymbol{r})$ and the solar radiation pressure acceleration $\boldsymbol{a}$ are defined by

$$
\begin{aligned}
U(\boldsymbol{r}) & =-\left[\frac{1}{2}|\boldsymbol{\omega} \times \boldsymbol{r}|^{2}+\frac{1-\mu}{r_{1}}+\frac{\mu}{r_{2}}\right], \\
\boldsymbol{a} & =a_{0}(\boldsymbol{S} \cdot \boldsymbol{n})^{2} \boldsymbol{n},
\end{aligned}
$$

where $\mu=0.1215$ is the mass ratio for the Earth-Moon system. The sail position vectors with respect to $m_{1}$ and $m_{2}$ respectively, are defined as $\boldsymbol{r}_{1}=[x+\mu, y, z]^{T}$ and $\boldsymbol{r}_{2}=[x-(1-\mu), y, z]^{T}$, and $a_{0}$ is the magnitude of the acceleration due to solar radition pressure exerted on the sail. The unit normal to the sail $\boldsymbol{n}$ and the Sun-line direction are given by

$$
\begin{aligned}
\boldsymbol{n} & =\left[\begin{array}{lll}
\cos (\gamma) \cos \left(\omega_{\star} t\right) & -\cos (\gamma) \sin \left(\omega_{\star} t\right) & \sin (\gamma)
\end{array}\right]^{T}, \\
\boldsymbol{S} & =\left[\begin{array}{lll}
\cos \left(\omega_{\star} t\right) & -\sin \left(\omega_{\star} t\right) & 0
\end{array}\right]^{T},
\end{aligned}
$$

where $\omega_{\star}=0.923$ is the angular rate of the Sun line in the corotating frame in a dimensionless synodic coordinate system. We will not consider the small annual changes in the inclination of the Sun-line with respect to the plane of the system.

\section{II.B. Linearized system}

We now investigate the dynamics of the sail in the neighborhood of the libration points. We denote the coordinates of the equilibrium point as $\boldsymbol{r}_{L}=\left(x_{L_{i}}, y_{L_{i}}, z_{L_{i}}\right)$ with $i=1, \cdots, 5$. Let a small displacement in $\boldsymbol{r}_{L}$ be $\delta \boldsymbol{r}$ such that $\boldsymbol{r} \rightarrow \boldsymbol{r}_{L}+\delta \boldsymbol{r}$. The equation of motion for the solar sail in the neighborhood of $\boldsymbol{r}_{L}$ are therefore

$$
\frac{d^{2} \delta \boldsymbol{r}}{d t^{2}}+2 \boldsymbol{\omega} \times \frac{d \delta \boldsymbol{r}}{d t}+\nabla U\left(\boldsymbol{r}_{L}+\delta \boldsymbol{r}\right)=\boldsymbol{a}\left(\boldsymbol{r}_{L}+\delta \boldsymbol{r}\right)
$$

Then, retaining only the first-order term in $\delta \boldsymbol{r}=[\xi, \eta, \zeta]^{T}$ in a Taylor-series expansion, where $(\xi, \eta, \zeta)$ are attached to the $L_{2}$ point as shown in Figure 1, the gradient of the potential and the acceleration can be expressed as

$$
\begin{aligned}
\nabla U\left(\boldsymbol{r}_{L}+\delta \boldsymbol{r}\right)= & \nabla U\left(\boldsymbol{r}_{L}\right)+\left.\frac{\partial \nabla U(\boldsymbol{r})}{\partial \boldsymbol{r}}\right|_{\boldsymbol{r}=\boldsymbol{r}_{L}} \delta \boldsymbol{r} \\
& +O\left(\delta \boldsymbol{r}^{2}\right) \\
\boldsymbol{a}\left(\boldsymbol{r}_{L}+\delta \boldsymbol{r}\right)= & \boldsymbol{a}\left(\boldsymbol{r}_{L}\right)+\left.\frac{\partial \boldsymbol{a}(\boldsymbol{r})}{\partial \boldsymbol{r}}\right|_{\boldsymbol{r}=\boldsymbol{r}_{L}} \delta \boldsymbol{r} \\
& +O\left(\delta \boldsymbol{r}^{2}\right)
\end{aligned}
$$

It is assumed that $\nabla U\left(\boldsymbol{r}_{L}\right)=0$, and the sail acceleration is constant with respect to the small displacement $\delta \boldsymbol{r}$, so that

$$
\left.\frac{\partial \boldsymbol{a}(\boldsymbol{r})}{\partial \boldsymbol{r}}\right|_{\boldsymbol{r}=r_{L}}=0
$$

The linear variational system associated with the libration points at $\boldsymbol{r}_{L}$ can be determined through a Taylor polynomial by substituting Eqs. (6) and (7) into (4)

$$
\frac{d^{2} \delta \boldsymbol{r}}{d t^{2}}+2 \boldsymbol{\omega} \times \frac{d \delta \boldsymbol{r}}{d t}-K \delta \boldsymbol{r}=\boldsymbol{a}\left(\boldsymbol{r}_{L}\right),
$$

where the matrix $K$ is defined as

$$
K=-\left[\left.\frac{\partial \nabla U(\boldsymbol{r})}{\partial \boldsymbol{r}}\right|_{\boldsymbol{r}=\boldsymbol{r}_{L}}\right]
$$

Using the matrix notation the linearized equation about the libration point (Equation (8)) can be represented

by the inhomogeneous linear system $\dot{\mathbf{X}}=A \mathbf{X}+\boldsymbol{b}(t)$, where the state vector $\mathbf{X}=(\delta \boldsymbol{r}, \delta \dot{\boldsymbol{r}})^{T}$, and $\boldsymbol{b}(t)$ is a $6 \times 1$ vector, which represents the solar sail acceleration. 
The Jacobian matrix $A$ has the general form

$$
A=\left(\begin{array}{cc}
0_{3} & I_{3} \\
K & \Omega
\end{array}\right)
$$

where $I_{3}$ is a identity matrix, and

$$
\Omega=\left(\begin{array}{rrr}
0 & 2 & 0 \\
-2 & 0 & 0 \\
0 & 0 & 0
\end{array}\right) .
$$

For convenience the sail attitude is fixed such that the sail normal vector $\boldsymbol{n}$, points always along the direction of the Sun-line with the following constraint $\boldsymbol{S} \cdot \boldsymbol{n} \geq 0$. Its direction is described by the pitch angle

$\gamma$ relative to the Sun-line, which represents the sail attitude. The linearized nondimensional equations of motion relative to a collinear libration point $L_{2}$ can then be written as

$$
\begin{aligned}
\ddot{\xi}-2 \dot{\eta}-U_{x x}^{o} \xi & =a_{\xi}, \\
\ddot{\eta}+2 \dot{\xi}-U_{y y}^{o} \eta & =a_{\eta}, \\
\ddot{\zeta}-U_{z z}^{o} \zeta & =a_{\zeta},
\end{aligned}
$$

where $U_{x x}^{o}, U_{y y}^{o}$, and $U_{z z}^{o}$ are the partial derivatives of the gravitational potential evaluated at the collinear libration point, and the solar sail acceleration is defined in terms of three auxiliary variables $a_{\xi}, a_{\eta}$, and $a_{\zeta}$

$$
\begin{aligned}
& a_{\xi}=a_{0} \cos \left(\omega_{\star} t\right) \cos ^{3}(\gamma), \\
& a_{\eta}=-a_{0} \sin \left(\omega_{\star} t\right) \cos ^{3}(\gamma), \\
& a_{\zeta}=a_{0} \cos ^{2}(\gamma) \sin (\gamma) .
\end{aligned}
$$

We will continue with the solution to the linearized equations of motion in the Earth-Moon restricted three-body problem in a later section.

\section{Moon-Sail Two-Body Problem}

In this section, we consider the motion of a solar sail moving under the gravitational influence of the Moon only as shown in Figure 2. Such a problem is defined as the Moon-Sail two-body problem. For a large displacement, such that the sail is far from the $L_{1}$ or $L_{2}$ point this provides a remarkably good approximation to the problem. The forces acting on the sail can be seen in Figure 3.

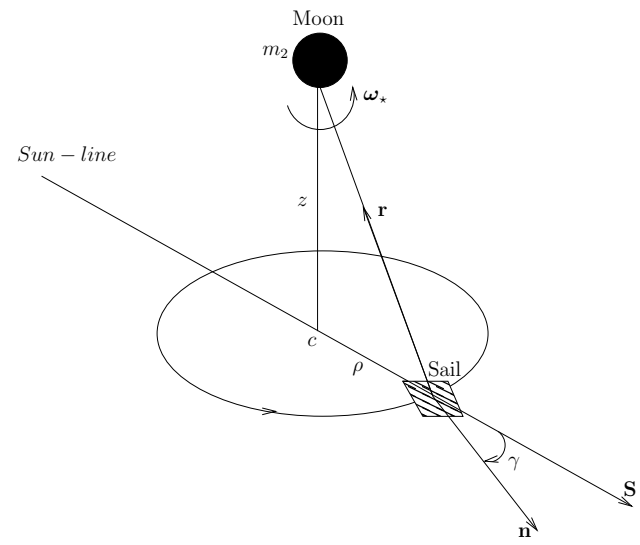

Figure 2. Schematic geometry of the MoonSail two-body problem generating a hover orbit.

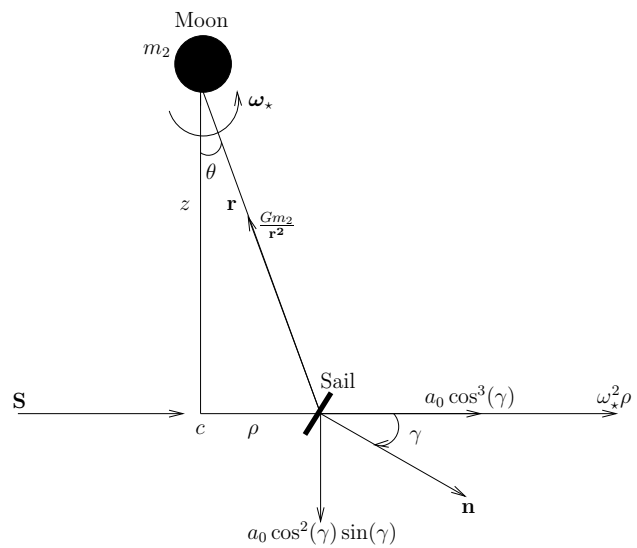

Figure 3. Representative forces. 


\section{III.A. Equations of motion}

In this model, the Moon is assumed to be fixed, while the solar sail is in a rotating frame of reference. To describe the motion of the sail, we take a reference frame rotating with the Sun-line at angular velocity $\boldsymbol{\omega}_{\star}$, such that the origin is at the center $c$. We write the two-body equations in a similar form to equation (2). In the rotating frame $\ddot{\boldsymbol{r}}=\dot{\boldsymbol{r}}=0$ and we have the equality

$$
\nabla \tilde{U}(\boldsymbol{r})=\boldsymbol{a},
$$

where

$$
\tilde{U}(\boldsymbol{r})=-\left[\frac{1}{2}\left|\boldsymbol{\omega}_{\star} \times \boldsymbol{r}\right|^{2}+\frac{G m_{2}}{r}\right] .
$$

The equations of motion of the solar sail in component form may be written in cylindrical coordinates $(\rho, z)$ as

$$
\begin{aligned}
\frac{G m_{2}}{r^{2}} \cos (\theta) & =a_{0} \cos ^{2}(\gamma) \sin (\gamma), \\
\frac{G m_{2}}{r^{2}} \sin (\theta)-\omega^{2} \rho & =a_{0} \cos ^{3}(\gamma),
\end{aligned}
$$

with $\cos (\theta)=\frac{z}{r}, \quad \sin (\theta)=\frac{\rho}{r}$, where $m_{2}$ is the mass of the Moon, $G$ is the gravitational constant and the distance of the solar sail from the Moon is $r=\sqrt{\rho^{2}+z^{2}}$ so that

$$
\begin{aligned}
\frac{G m_{2} z}{r^{3}} & =a_{0} \cos ^{2}(\gamma) \sin (\gamma) \\
\frac{G m_{2} \rho}{r^{3}} & =a_{0} \cos ^{3}(\gamma)+\omega^{2} \rho
\end{aligned}
$$

Rearranging the equations (22) and (23), we obtain

$$
\tan (\gamma)=\frac{z}{\rho}\left[1-\left(\frac{\omega}{\tilde{\omega}}\right)^{2}\right]^{-1}
$$

for a given $(\rho, z)$, where

$$
\tilde{\omega}^{2}=\frac{G M}{r^{3}} .
$$

Similary from equations (22) and (23), the required radiation pressure acceleration for the two-body analysis may also be obtained as

$$
a_{0}=\cos ^{2}(\gamma)^{-1}\left[\left(\left(\tilde{\omega}^{2} z\right)^{2}+\left(\tilde{\omega}^{2} \rho-\omega^{2} \rho\right)^{2}\right]^{1 / 2} .\right.
$$

We now have conditions for a large displaced periodic orbit centered on the Moon. We will evaluate the usefullness of this model later.

\section{Solution of the linearized equations of motion for the three-body model}

In order to evaluate the two and three-body models, we will obtain a displaced periodic orbit from the linearized dynamics defined earlier.

Considering the dynamics of motion near the collinear libration points, we may choose a particular periodic solution in the plane of the form (see $\operatorname{Farquhar}^{24}$ )

$$
\begin{aligned}
& \xi(t)=\xi_{0} \cos \left(\omega_{\star} t\right), \\
& \eta(t)=\eta_{0} \sin \left(\omega_{\star} t\right) .
\end{aligned}
$$


By inserting equations (27) and (28) in the differential equations (12-14), we obtain the linear system in $\xi_{0}$ and $\eta_{0}$,

$$
\left\{\begin{array}{c}
\left(U_{x x}^{o}-\omega_{\star}^{2}\right) \xi_{0}-2 \omega_{\star} \eta_{0}=a_{0} \cos ^{3}(\gamma), \\
-2 \omega_{\star} \xi_{0}+\left(U_{y y}^{o}-\omega_{\star}^{2}\right) \eta_{0}=-a_{0} \cos ^{3}(\gamma) .
\end{array}\right.
$$

Then the amplitudes $\xi_{0}$ and $\eta_{0}$ are given by

$$
\begin{aligned}
& \xi_{0}=a_{0} \frac{\left(U_{y y}^{o}-\omega_{\star}^{2}-2 \omega_{\star}\right) \cos ^{3}(\gamma)}{\left(U_{x x}^{o}-\omega_{\star}^{2}\right)\left(U_{y y}^{o}-\omega_{\star}^{2}\right)-4 \omega_{\star}^{2}}, \\
& \eta_{0}=a_{0} \frac{\left(-U_{x x}^{o}+\omega_{\star}^{2}+2 \omega_{\star}\right) \cos ^{3}(\gamma)}{\left(U_{x x}^{o}-\omega_{\star}^{2}\right)\left(U_{y y}^{o}-\omega_{\star}^{2}\right)-4 \omega_{\star}^{2}},
\end{aligned}
$$

and we have the equality

$$
\frac{\xi_{0}}{\eta_{0}}=\frac{\omega_{\star}^{2}+2 \omega_{\star}-U_{y y}^{o}}{-\omega_{\star}^{2}-2 \omega_{\star}+U_{x x}^{o}} .
$$

The trajectory will therefore be an ellipse centered on a collinear libration point. We can find the required radiation pressure acceleration by solving equation (30)

$$
a_{0}=\cos ^{-3}(\gamma)\left[\frac{\omega_{\star}^{4}-\omega_{\star}^{2}\left(U_{x x}^{o}+U_{y y}^{o}+4\right)+U_{x x}^{o} U_{y y}^{o}}{U_{y y}^{o}-2 \omega_{\star}-\omega_{\star}^{2}}\right] \xi_{0} .
$$

By applying the Laplace transform, the uncoupled out-of-plane $\zeta$-motion defined by the equation (14) can be obtained as

$$
\zeta(t)=U(t) a_{0} \cos ^{2}(\gamma) \sin (\gamma)\left|U_{z z}^{o}\right|^{-1}+\dot{\zeta}_{0}\left|U_{z z}^{o}\right|^{-1 / 2} \sin \left(\omega_{\zeta} t\right)+\cos \left(\omega_{\zeta} t\right)\left[\zeta_{0}-a_{0} \cos ^{2}(\gamma) \sin (\gamma)\left|U_{z z}^{o}\right|^{-1}\right.
$$

where the nondimensional frequency $\omega_{\zeta}$ is defined as

$$
\omega_{\zeta}=\left|U_{z z}^{o}\right|^{1 / 2}
$$

and $U(t)$ is the unit step function.

A sufficient condition for displaced orbits based on the sail pitch angle $\gamma$ and the magnitude of the solar radiation pressure $a_{0}$ for fixed initial out-of-plane distance $\zeta_{0}$ can be derived. Specifically for the choice of the initial data $\dot{\zeta}_{0}=0$, equation (33) can be more conveniently expressed as

$$
\begin{aligned}
\zeta(t)= & U(t) a_{0} \cos ^{2}(\gamma) \sin (\gamma)\left|U_{z z}^{o}\right|^{-1} \\
& +\cos \left(\omega_{\zeta} t\right)\left[\zeta_{0}-a_{0} \cos ^{2}(\gamma) \sin (\gamma)\left|U_{z z}^{o}\right|^{-1}\right] .
\end{aligned}
$$

The solution can then be made to contain only a constant displacement at an out-of-plane distance

$$
\zeta_{0}=a_{0} \cos ^{2}(\gamma) \sin (\gamma)\left|U_{z z}^{o}\right|^{-1}
$$

Furthermore, the out-of-plane distance can be maximized by an optimal choice of the sail pitch angle determined by

$$
\begin{aligned}
\frac{d}{d \gamma^{\star}} \cos ^{2}\left(\gamma^{\star}\right) \sin \left(\gamma^{\star}\right) & =0, \\
\gamma^{\star} & =35^{\circ} .264 .
\end{aligned}
$$

We now have conditions for a small displaced periodic orbit centered on the collinear libration points. 


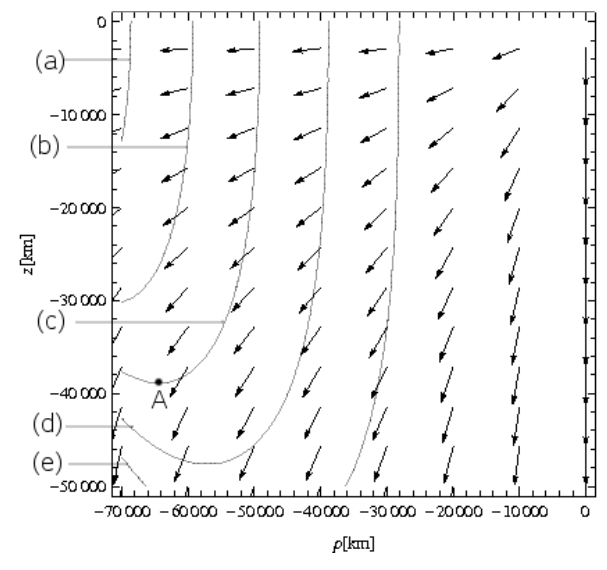

Figure 4. A contour plot and the vector field of the characteristic acceleration $a_{0}=$ $a_{0}(\rho, z)$ in the Earth-Moon system: (a) $a_{0}=$ $0.58 \mathrm{~mm} / \mathrm{s}^{2}$, (b) $a_{0}=1 \mathrm{~mm} / \mathrm{s}^{2}$, (c) $a_{0}=1.7$ $\mathrm{mm} / \mathrm{s}^{2}$, (d) $a_{0}=3 \mathrm{~mm} / \mathrm{s}^{2}$, (e) $a_{0}=6 \mathrm{~mm} / \mathrm{s}^{2}$.

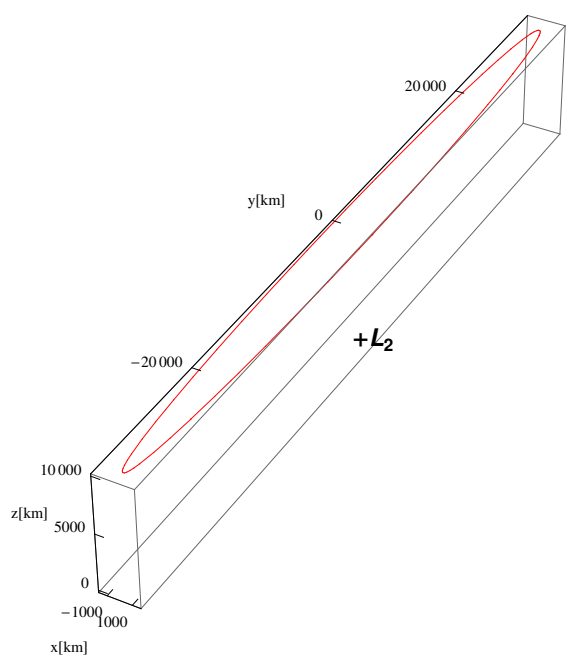

Figure 5. Linear analysis for small $a_{0}=0.58$ $\mathrm{mm} / \mathrm{s}^{2}$ (Orbit around $L_{2}$ ).

\section{Comparison of the linear three-body and the approximate two-body solution}

In this section, we compare the dynamics near the Earth-Moon $L_{1}$ and $L_{2}$ points for small accelerations with the linear analysis, and the large hover orbits for large acceleration with the two-body analysis to the orbit found by Ozimek et al. ${ }^{17}$ using a full three-body analysis. We demonstrate that for a given orbit radius $\rho$ and displacement distance $z$, we can find the characteristic acceleration $a_{0}$ and the sail pitch angle $\gamma$ using the two-body analysis.

Let us consider the vector field on the plane given by

$$
\boldsymbol{n}(\rho, z)=\left(\frac{f_{2}(\rho, z)}{\sqrt{f_{1}^{2}(\rho, z)+f_{2}^{2}(\rho, z)}}, \frac{f_{1}(\rho, z)}{\sqrt{f_{1}^{2}(\rho, z)+f_{2}^{2}(\rho, z)}}\right),
$$

where

$$
\begin{aligned}
f_{1}(\rho, z) & =\frac{G m_{2} z}{r^{3}}, \\
f_{2}(\rho, z) & =\frac{G m_{2} \rho}{r^{3}}-\omega^{2} \rho .
\end{aligned}
$$

For a given $(\rho, z)$ the contours of Figure 4 define the require sail accelaration $a_{0}$ from equation (26) while the vector field describes the required sail orientation $\boldsymbol{n}$. From equations (22) and (23), this vector field defined on the whole plane minus the origin describes the direction of the acceleration vector $\boldsymbol{a}=\boldsymbol{a}(\rho, z)$.

This figure indicates for a large characteristic acceleration $a_{0}=1.7 \mathrm{~mm} / \mathrm{s}^{2}$ an orbit with radius $\rho \approx 6 \times 10^{4}$ $\mathrm{km}$ and displacement distance $z \approx 4 \times 10^{4} \mathrm{~km}$ is possible. The point marked A in Figure 4 represents the optimal displaced orbit. A small orbit at $L_{2}$ with characteristic acceleration $a_{0}=0.58 \mathrm{~mm} / \mathrm{s}^{2}$ is shown in Figure 5 using the linear analysis.

Near $L_{1}$ and $L_{2}$ the displacement distance for the linear analysis for a small acceleration $a_{0}=0.58 \mathrm{~mm} / \mathrm{s}^{2}$ and the two-body analysis for large acceleration $a_{0}=1.7 \mathrm{~mm} / \mathrm{s}^{2}$ give a good approximation to the orbits found by Ozimek et al. ${ }^{17}$ using a full three-body analysis (see Table 1 ). 
Table 1. Comparison of displacement distance and orbit radius.

\begin{tabular}{lcccc}
$a_{0}\left(\mathrm{~mm} / \mathrm{s}^{2}\right)$ & $z(\mathrm{~km})^{a}$ & $z(\mathrm{~km})^{b}$ & $\rho(\mathrm{km})^{a}$ & $\rho(\mathrm{km})^{b}$ \\
\hline 0.58 & $\approx 10^{4}$ & $\approx 10^{4^{*}}$ & - & - \\
1.70 & $\approx 4.5 \times 10^{4}$ & $\approx 4.0 \times 10^{4 \dagger}$ & $\approx 5.6 \times 10^{4}$ & $\approx 6.5 \times 10^{4}$
\end{tabular}

${ }^{a}$ Displacement and radius found by Ozimek et al. using Hermite-Simpson and seventh-degree Gauss Lobatto collocation schemes ${ }^{17}$.

${ }^{\mathrm{b}}$ Displacement and radius found by the approximate analysis in this paper.

* Displacement distance obtain from the linear analytical solution.

$\dagger$ Displacement distance obtain from the two-body approximation.

\section{Conclusion}

This paper has demonstrated the approximation of large displaced orbits in the Earth-Moon circular restricted three-body problem by the Moon-Sail two-body problem. In addition, based on the linearized equation of motion near the collinear Lagrange points, displaced periodic orbits can be approximated by using linear analytical analysis, while far from those points the classical two-body problem gives a good approximation. A sufficient condition for displaced periodic orbits based on the sail pitch angle and the magnitude of the solar radiation pressure for fixed initial out-of-plane distance has been derived. It was shown that for a given orbit radius and displacement distance, we can find the characteristic acceleration and the sail pitch angle using the two-body analysis. The orbits found approach the asymptotic solutions as the characteristic acceleration becomes large. A particular use of such orbits include continous communications between the equatorial regions of the Earth and the lunar poles.

\section{Acknowledgments}

This work was funded by the European MCRTN (Marie Curie Research Training Network) AstroNet, Contract Grant No. MRTN-CT-2006-035151.

\section{References}

${ }^{1}$ McInnes, C. R., Solar sailing: technology, dynamics and mission applications, Springer Praxis, London, 1999, pp. 11-29.

${ }^{2}$ Waters, T. and McInnes, C., "Periodic Orbits Above the Ecliptic in the Solar-Sail Restricted Three-Body Problem," J. of Guidance, Control, and Dynamics, Vol. 30, No. 3, 2007, pp. 687-693.

${ }^{3}$ Forward, R. L., "Statite: A Spacecraft That Does Not Orbit," Journal of Spacecraft and Rocket, Vol. 28, No. 5, 1991, pp. 606-611.

${ }^{4}$ Szebehely, V., Therory of Orbits: the restricted problem of three bodies, Academic Press, New York and London, 1967, pp. 497-525.

${ }^{5}$ Roy, A. E., Orbital Motion, Institute of Physics Publishing, Bristol and Philadelphia, 2005, pp. 118-130.

${ }^{6}$ Vonbun, F., "" A Humminbird for the $\mathrm{L}_{2}$ Lunar Libration Point"," Nasa TN-D-4468, April 1968.

${ }^{7}$ Thurman, R. and Worfolk, P., "The geometry of halo orbits in the circular restricted three-body problem," Technical report GCG95, Geometry Center, University of Minnesota, 1996.

${ }^{8}$ Gómez, G., Llibre, J., Martínez, R., and Simó, C., Dynamics and Mission Design Near Libration Points, Vol. II, World Scientific Publishing Co.Pte.Ltd, Singapore.New Jersey.London.Hong Kong, 2001, Chaps. 1, 2.

${ }^{9}$ Gómez, G., Jorba., A., J.Masdemont, and Simó, C., Dynamics and Mission Design Near Libration Points, Vol. IV, World Scientific Publishing Co.Pte.Ltd, Singapore.New Jersey.London.Hong Kong, 2001, Chap. 2.

${ }^{10}$ Farquhar, R. and Kamel, A., "Quasi-periodic orbits about the trans-lunar libration point," Celestial Mechanics, Vol. 7, 1973, pp. 458-473.

${ }^{11}$ Farquhar, R., "The utilization of Halo orbits in advanced lunar operations," Nasa technical report, 1971.

${ }^{12}$ Breakwell, J. and Brown, J., "The 'halo' family of 3-dimensional periodic orbits in the Earth-Moon restricted 3-body problem," Celestial Mechanics, Vol. 20, 1979, pp. 389-404.

${ }^{13}$ Richardson, D. L., "Halo orbit formulation for the ISEE-3 mission," J. Guidance and Control, Vol. 3, No. 6, 1980, pp. $543-548$.

${ }^{14}$ Howell, K., "Three-dimensional, periodic, 'halo' orbits," Celestial Mechanics, Vol. 32, 1984, pp. 53-71.

${ }^{15}$ Howell, K. and Marchand, B., "'Natural and Non-Natural Spacecraft Formations Near $\mathrm{L}_{1}$ and L Libration Points in the Sun-Earth/Moon Ephemerics System"," Dynamical Systems: An International Journal, Vol. 20, No. 1, March 2005, pp. 149-173. 
${ }^{16}$ McInnes, C., "Solar sail Trajectories at the Lunar L2 Lagrange Point," J. of Spacecraft and Rocket, Vol. 30, No. 6, 1993, pp. $782-784$.

${ }^{17}$ Ozimek, M., Grebow, D., and Howell, K., "Solar Sails and Lunar South Pole Coverage," In AIAA/AAS Astrodynamics Specialist Conference and Exhibit, AIAA Paper 2008-7080, Honolulu, Hawaii, August 2008.

${ }^{18}$ Baoyin, H. and McInnes, C., "Solar sail halo orbits at the Sun-Earth artificial $\mathrm{L}_{1}$ point," Celestial Mechanics and Dynamical Astronomy, Vol. 94, No. 2, 2006, pp. 155-171.

${ }^{19}$ Baoyin, H. and McInnes, C., "Solar sail equilibria in the elliptical restricted three-body problem," Journal of Guidance, Control and Dynamics, Vol. 29, No. 3, 2006, pp. 538-543.

${ }^{20}$ Baoyin, H. and McInnes, C., "Solar sail orbits at artificial Sun-Earth Lagrange points," Journal of Guidance, Control and Dynamics, Vol. 28, No. 6, 2005, pp. 1328-1331.

${ }^{21}$ McInnes, C. R., "Artificial Lagrange points for a non-perfect solar sail," Journal of Guidance, Control and Dynamics, Vol. 22, No. 1, 1999, pp. 185-187.

${ }^{22}$ McInnes, C., McDonald, A., Simmons, J., and McDonald, E., "Solar sail parking in restricted three-body systems," Journal of Guidance, Control and Dynamics, Vol. 17, No. 2, 1994, pp. 399-406.

${ }^{23}$ Simo, J. and McInnes, C. R., "Solar sail trajectories at the Earth-Moon Lagrange points," In 59th International Astronautical Congress, IAC Paper 08.C1.3.13, Glasgow, Scotland, 29 September - 03 October 2008.

${ }^{24}$ Farquhar, R., "The Control and Use of Libration-Point Satellites," Ph.D. Dissertation, Stanford University, 1968. 